\title{
Dynamic Analysis of a Convergence Diagnostic Equipment for Preventive Maintenance in Light Vehicles
}

\author{
Claudio Cordova ${ }^{1}$, Fredy Llulluna ${ }^{2}$, Juan Sinchi $^{3}$, David Pachacama ${ }^{4}$, Darwin Pachacama ${ }^{5}$ \\ ${ }^{1}$ Department of Basic Sciences /Escuela Politécnica Nacional \\ Ladrón de Guevara E11-253, Quito, Ecuador \\ claudio.cordova@epn.edu.ec \\ ${ }^{2}$ Deparament Sustainable Architecture /Universidad Regional Amazónica Ikiam \\ Parroquia Muyuna, kilómetro 7 vía a Alto Tena. Napo, Ecuador \\ fredy.llulluna@ikiam.edu.ec \\ ${ }^{3}$ Deparament Automotive Engineering / Universidad Politécnica Salesiana \\ Calle Vieja 12-30 y Elia Liu, Cuenca, Ecuador \\ jsinchi@ups.edu.ec \\ ${ }^{4,5}$ Deparament of Exacts Sciences / Universidad de las Fuerza Armadas ESPE \\ Av. Gral. Rumiñahui y Av. Ambato, Sangolquí, Ecuador \\ dppachacama1@espe.edu.ec/dapachacama@espe.edu.ec
}

\begin{abstract}
This research carries out the dynamic analysis of light and semi-light automotive convergence diagnostic equipment, with a gross weight of up to three tons. A comparative analysis of the implications and consequences due to positive, negative, and neutral convergences is carried out. Then the different mechanical and electronic design characteristics of the device to determine the convergence in the tires. The design of the equipment has three stages: mechanical, electrical, and electronic. The mechanical stage comprises the structure of the equipment, the electrical stage consisting of the design and construction of a longitudinal motion sensor, The electronic stage formed by a microcontroller which, once the microprocessor has been programmed, performs the measurement process based on the variables: relative displacement and potential difference. The elements designed and built are integrated into the equipment, which through a test protocol was evaluated both in empty and under load.
\end{abstract}

Keywords: dynamic, electromechanical, convergence, automotive, alignment.

\section{Introduction}

The good mechanical condition of the vehicle, and the correct calibration of each of its parts or systems, ensure good dynamic response and safety of its occupants. Each year about 1.3 million people on the roads of the world, between 20 and 50 million suffer serious and minor injuries. Traffic accidents are one of the main causes of death in all age groups, and the first among people between 15 and 29 years of age (UN, 2019). In addition to economic losses, and psychological consequences of people who have suffered after a car accident. These reasons are enough to understand that developed the automotive industry over time has put emphasis on energy efficiency, operational functionality, aesthetics, safety, and minimizing the harmful effects to the environment caused as a result of its functioning.

The parameters that reflect a good dynamic response of the vehicle are caster or advance, camber or fall, convergence or divergence of its tires, the inclination of ball joints, thrust angle, and inclination of the steering axis, among others. [1] In the present work, convergence alignment is studied in automobiles with a maximum weight of $4000 \mathrm{~kg}$. The misalignment of convergence of the vehicle is classified as a serious defect in the dynamic behavior of the same according to the guidelines of the Vehicle Technical Review implemented in our country at the national level, it considers that any defect of the vehicle classified as serious, must be corrected compulsorily movement in urban districts. These regulations must be complied with for the annual certificate of good vehicle operation to be issued. The main objective is to design and build a two-part diagnostic kit, to detect toe misalignment in a vehicle and perform the measurement to correct such a defect. The alignment equipment is scarce, and the cost is high, the present work is an alternative for local development, with a view to national industrial development. 
Convergence is measured in degrees and the measurement is performed from the rearmost of the rim to the height of the cube and most forward point. The difference between the two measurements determines the toe-in value that indicates what, precisely, the type of toe-in of the wheels. The angle between the plane that contains the lateral faces of the rim and the vertical plane concerning the ground (horizontal). This camber or fall angle in sports cars can measure up to $5^{\circ}$, in motorcycles it can reach $50^{\circ}$. [3] For four-wheel vehicles, the camber angle can be understood as follows: if the planes containing the pair of fronts (or rear) tires are parallel to each other and perpendicular to the ground, the camber is said to be zero. The planes containing the pair of fronts (or rear) tires intersect upward from the horizontal floor, forming an acute angle with each other, the camber angle is said to be negative, and its magnitude is the number of degrees the camber deviates a plane containing the lateral face of the rim for the vertical plane for the ground. If the planes containing the pair of fronts (or rear) tires intersect below the horizontal floor, forming an acute angle with each other, the camber angle is said to be positive, and its magnitude is the number of degrees the plane deviates. It contains the lateral face of the tire for the vertical plane to the ground. [4]

If the planes containing the pair of fronts (or rear) tires intersect towards the front of the vehicle, forming an acute angle between them, the vehicle tires are said to have positive toe-in and their magnitude is given by the difference between the front and rear parts of the pair of front tires (or the pair of rear tires), in other words. When the front of the wheels is closer to each other than their respective backs, these wheels are said to be convergent. When the front parts of the wheels are further apart than their respective rear parts, this pair of wheels is said to diverge or to be divergent. [4] [5]

\section{Convergence and Its Effects}

The torque or torque of the torque transmitted from the engine to the wheels of the vehicle, allowing the rotation of the tires and drive the vehicle. In Figure 1, the torque shown $\vec{F},-\vec{F}$, friction force $\vec{f}_{r}$ between the tire and the ground and the movement of the axis of the tire (vehicle movement) with speed $\vec{v}$.

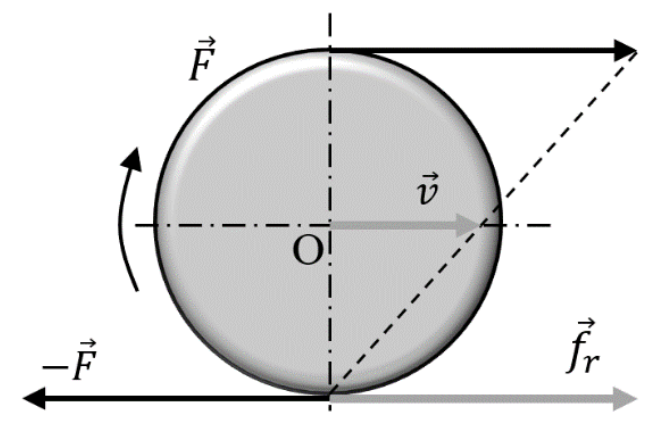

Fig. 1: Race Cars Vehicle Dynamics. [4]

The force $-\vec{F}$ and the component $\vec{F}$, cancels out with friction force $\vec{f}_{r}$ between the wheel and the floor, then the force $\vec{F}$ is the cause of the relative movement of the wheel with respect to the floor, thus, the axis of the wheel will move with respect to the floor with speed $\vec{v}$. Considering that the wheel axis is subject to the chassis of the vehicle, then its movement relative to the floor is the movement of the vehicle relative to the floor. The friction force between the wheel and the floor can change with the type of tire, the type of floor, the weight of the vehicle, the inflation pressure, the tire wear. If the friction force fails to nullify the force $-\vec{F}$ of the pair, then the tire starts to slide or skid decreasing mobility of the drive shaft, which connects the forward motion of the vehicle relative to the floor. [6]

This section presents the concepts and definitions of convergence; null, positive and negative, and their repercussions, vital when designing and building a convergence diagnostic team. All the diagrams presented illustrate the movement of the front left tire, bearing in mind that the movement of the front right rim is symmetrical to the left rim concerning the vehicle's advance line. Figure 2a illustrates the movement of wheel A, so it rotates with angular velocity w, advancing towards the front of the vehicle, driven by the engine $\mathrm{M}$. The centerline I of the rim is superimposed on line R, by the one that moves the vehicle forward. Then the degree of toe-in of the tire is said to be zero or zero. [7] Under these conditions, if the tire treads 
and turns on a sheet of paper lying on the ground and if there is no friction between the sheet and the ground, the sheet of paper moves in the $\mathrm{H}$ direction, aligned with the centerline I of the rim. The paper does not suffer any lateral deviation. The vehicle moves in the forward direction even if the driver takes their hands off the wheels.

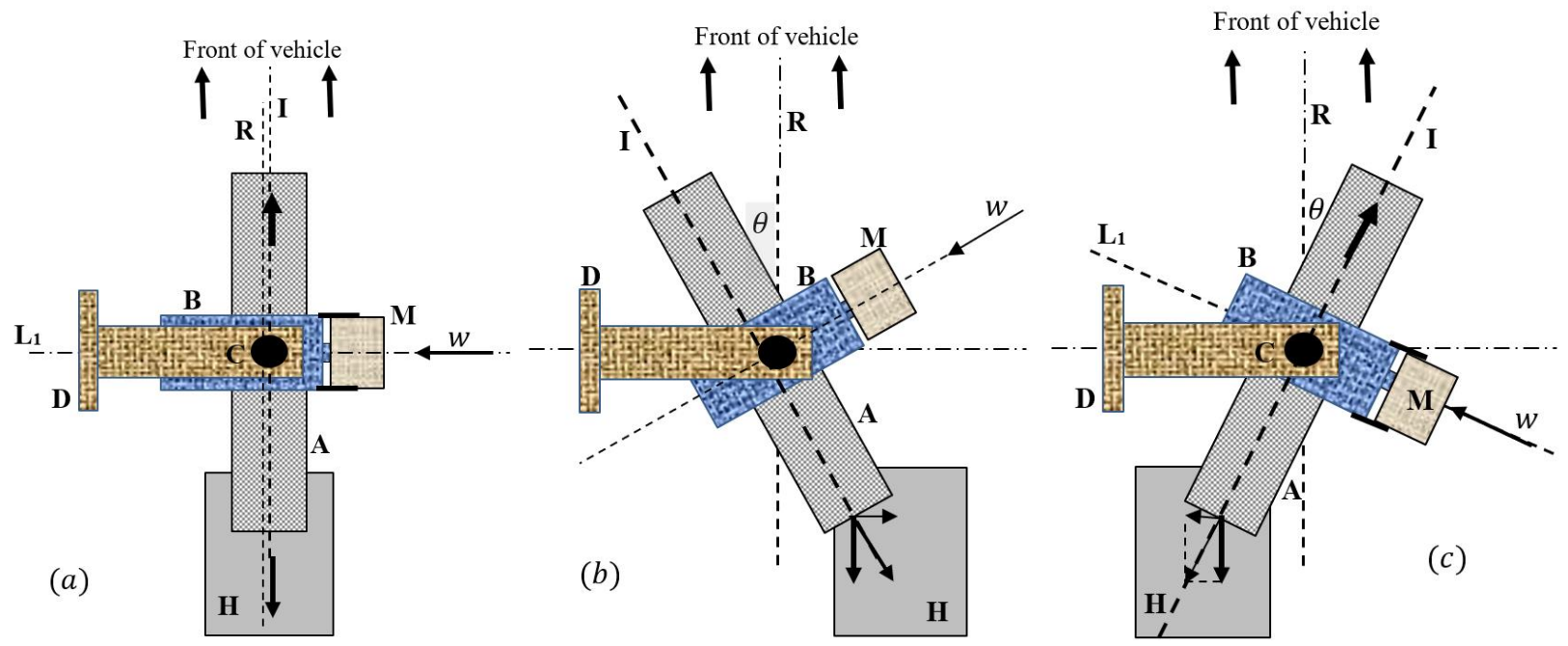

Fig. 2: Null convergence (a), negative convergence (b), positive convergence

Negative convergence or divergence occurs when the movement of the left front wheel A (the orientation of the right front wheel is symmetrical to the left, concerning the line R), so that it rotates with angular velocity w, driven by the motor $\mathrm{M}$ with the centerline I of the wheel angle $\theta$ deviated one in the anti-clockwise, to the line direction $\mathrm{R}$ in which the vehicle moves forward, 2a. Then it is understood that the convergence of the tire is negative and different from zero.

The positive convergence or just convergence is observed in the movement of the left front wheel A. The orientation of the right front wheel is symmetrical to the left relative to the line $\mathrm{R}$ by advancing the vehicle, it rotates with angular velocity $\mathrm{w}$, driven by the motor $\mathrm{M}$. The centerline I of the wheel is offset by an angle $\theta$ in the clockwise direction, concerning the direction line $\mathrm{R}$ in which the vehicle is moving forward. It is then said that convergence of the rim is positive and different from zero, Figure 1c. Positive convergence is commonly referred to simply as convergence.

\subsection{Displacements Generated By Misalignment}

When the vehicle is forced to advance on a line other than the centerline of the rim, with its tires in a positive or negative toe-in condition, large forces are generated on the tire pivot, which is the axis around which they are oriented. On the other hand, the movement of the vehicle along the line of advance is difficult, this opposition to moving along the line of advance is greater the degree of misalignment. This proportionality is not a linear relationship.

The convergence alignment values different from zero, also generate forces concentrated at the edges of the tires (especially the inner edges), causing rapid wear thereof. A convergence nonzero in the rear wheels in addition to the rapid wear of the tire can change the angle of the motor thrust. The convergence alignment values are positive or negative and can be corrected by adjusting the threaded ends that connect the rim to the rod. [9]

An indicator of the convergence value is the value of the smallest angle formed between the vehicle's line of advance and the center line of the rim, which at most, rarely reaches a value of two degrees, therefore, its adjustment does not It can be done by relying on the direct perception of the eye but requires a precision instrument. This detail justifies the objective of working on the design and construction of a convergence diagnostic equipment that allows correcting the misalignment defect of automotive tires. 


\section{Methodology}

The cases of convergence positive, null, and negative toe-in respectively for the same left front tire of the car. In all cases, the vehicle is forced to move in the same direction R, even though in cases: a), c), its tires deviate from the direction of advance as shown by the centerline I of the tire. In all cases the wheel rotates with an angular velocity, wy magnitude perpendicular to the plane of relative rotation, then, if the rim in its movement, treads friction to a sheet of paper which in turn slides frictionless relative to the floor, it is displaced by drag in the direction $\vec{H}$ different in each case as shown in this figure 3. Each displacement $\vec{H}$ can be decomposed into its Cartesian components: $\vec{H}_{x}, \vec{H}_{y}$. In all the cases shown, the displacements $\vec{H}_{y}$ occur in the same direction, so these cannot be used as indicators of the different forms of convergence alignment, however, the lateral displacements $\vec{H}_{x}$ generate the difference between the different cases of convergence alignment, like this: a positive convergence produces a lateral displacement of the paper sheet $\vec{H}_{x}$ to the left, a null convergence produces a null displacement $\vec{H}_{x}$ and a negative convergence produces a lateral displacement $\vec{H}_{x}$ towards the right of the sheet of paper. It is for this reason that the lateral displacements $\vec{H}_{x}$ of the sheet of paper constitute in themselves adequate indicators of the different cases of convergence alignment.

The movement of the sheet dragged by the wheels is 2-dimensional, its movement is said to have two degrees of freedom. On the other hand since the displacement $\vec{H}_{y}$. of the blade does not provide information on the different forms of alignment, it can be blocked and information on the convergence alignment of the rim is not lost. Consequently, to have the information of convergence of the rim, to skip the displacement $\vec{H}_{y}$. In this sense, it is said that a degree of freedom of the movement of the blade has been restricted, resulting in that, to determine the different forms of convergence, only the lateral displacement $\vec{H}_{x}$ of the blade dragged by the rim through friction must be carefully observed.

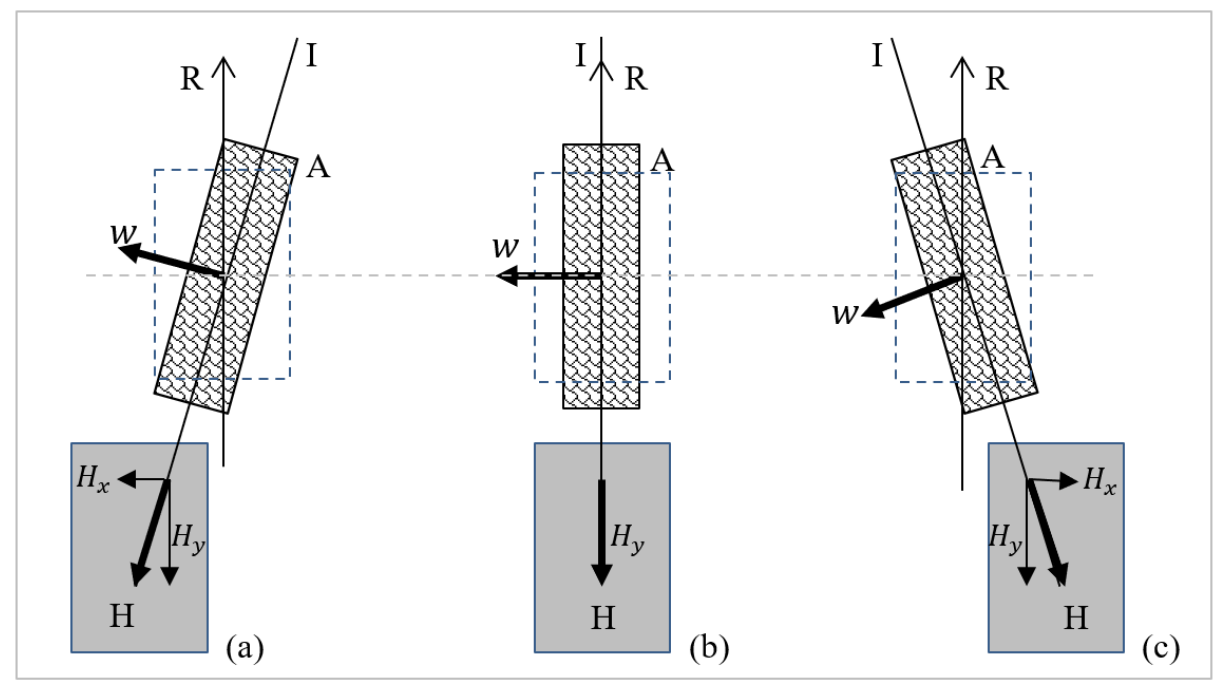

Fig. 3: Degrees of freedom on the wheels

El The study of the movement of the sheet of paper, displaced by friction by the rim and its lateral sliding on the smooth horizontal floor, is the focus of attention to investigate and quantify the different effects that occur in the convergence alignment, and constitutes the conceptual basis for the development of diagnostic equipment for convergence and can be abstracted as follows. The sheet of paper can be replaced by a steel plate, with one of its faces rough and the other smooth, this characteristic can be obtained by adhering a mechanical anti-friction system to the lower face of the plate. Once its movement is restricted in the vehicle's line of advance, its lateral displacement $\vec{H}_{x}$, perpendicular to the line of advance, will give information about the convergence alignment. In fact, building such a mechanism is possible and constitutes an essential first element of our alignment team. The second essential element is to solve the problem of quantization of the lateral 
movement of the steel deck, taking into account the value of lateral displacement is very small relative to the forward travel of the vehicle. Simple calculations show that the accuracy of the transverse displacement measurement $\left|\vec{H}_{x}\right|$ is a millimeter or smaller in magnitude.

\subsection{Desarrollo De Equipo A Nivel Conceptual}

Mechanical stage in its conceptual level, coupled the idea of the paper sheet by the tire tread, with one side rubbing against the rim and the other sliding without friction relative to the floor. The mechanical stage highlights three sections: the mechanical section 1.1 Simulates the fixed ground floor. The mechanical section 1.2 idealizes smooth, between the lower face of the paper sheet and the floor. The mechanical section 1.3 idealizes the movement of the sheet relative to ground, smooth on its lower face and roughened on its upper face.

The conceptual level of the electrical stage of the equipment requires an element that can transform a displacement vector into an associated voltage measurement, this type of element, Figure 6 is shown, represented by a transformer block, with an input variable the displacement and an output variable such as the associated voltage, called (according to Soria, 2013) an open-loop system. We call this element a motion sensor. The conceptual level of the electronic stage is outlined in figure 4. A system is required that allows obtaining a set of data, processing it, managing it, and proceeding to display it..

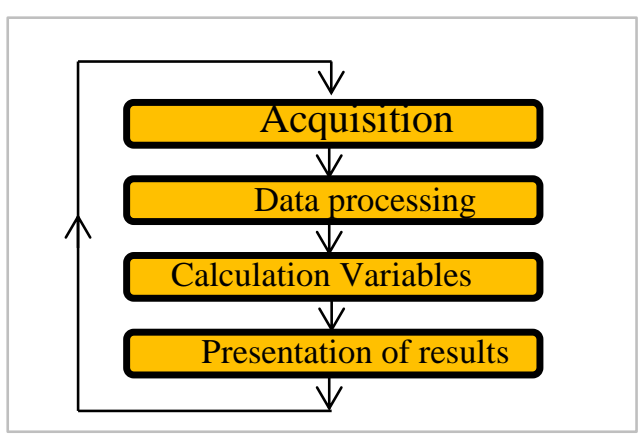

Fig. 4: Diagram of the convergence system

\subsection{Development of the convergence alignment device}

Mechanical stage, basic development. The component $\vec{H}_{y}$. of the displacement. does not differentiate the different types of convergence, its restriction does not affect the value of the indicator $\vec{H}_{x}$, Figure 4a illustrates the blocking of the longitudinal movement $\vec{H}_{y}$ of section 1.3 concerning section 1.1. However, its transverse rectilinear motion Hx is free. In this figure: section 1.1 is anchored to the floor; the intermediate section 1.2 formed by a surface distribution of antifriction spheres allow a frictionless movement of 1.3 concerning section 1.1; The "V" shaped channels made in the sidebars of sections 1.1 and 1.3 prevent their relative longitudinal displacement; The arrow at the top of Figure 5 is the vehicle's direction R of advance. The value of transverse displacement $\vec{H}_{x}$., is proportional to the value of the convergence defect,

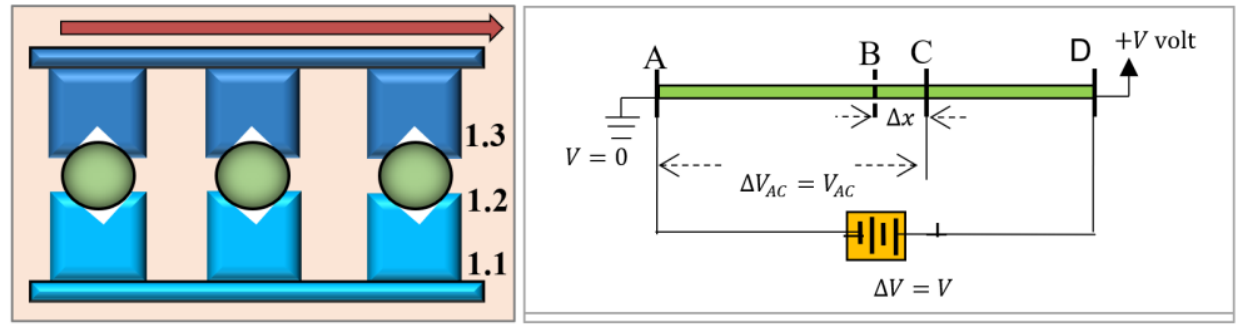

Fig. 5: Mechanical and electronic diagram 
Electrical stage, basic development, constitutes the step from the ideas shown in figure $4 \mathrm{~b}$ to the concrete ideas shown in figure 4, where the transformer block is constituted by a movable cursor potentiometer $\mathrm{B}$, free to move between its ends A, D.

Electronic stage, basic development, can be seen in figure 10, in it, three sub-stages are seen: A, B, C. In stage A, the input voltage for the RV1 motion sensor is conditioned, and its signal output enters the microcontroller, in this stage B, the microcontroller must achieve the measurement system and process it, it must calculate variables involved in the convergence phenomenon, and then in stage $\mathrm{C}$ display them on the screen, figure 6.

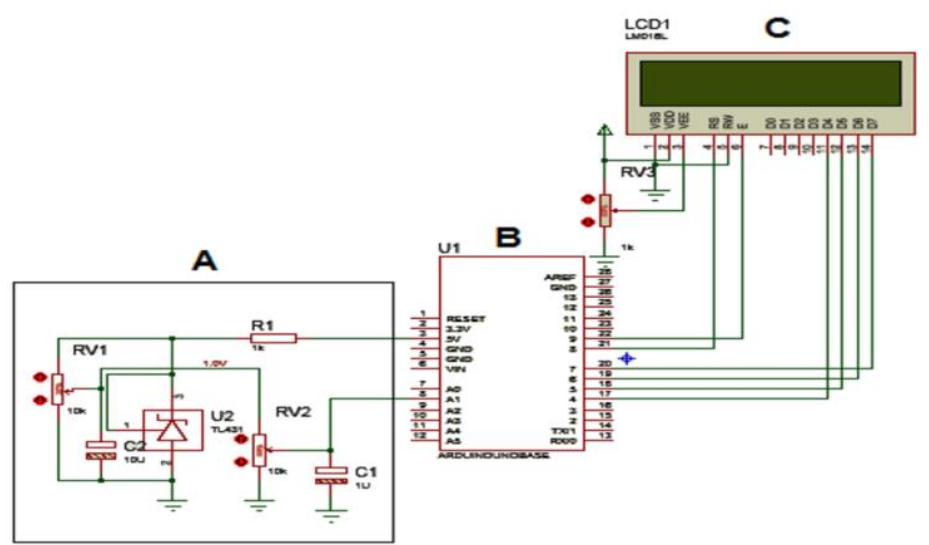

Table 6: Microcontroller Diagram

\subsection{Mechanical Design of the Structure}

El The overall design of the assembly can be seen in figure 11 . The sections and their corresponding elements are Meshshaped support structure, ribbed crossbars, reciprocating elastic mechanism, movement sensor, mechanical waxer, safety pillars, vertical movement blockers. Mobile elements: layette rectilinear, transverse , 49 bearing areas , 19mm diameter and finally top cover.
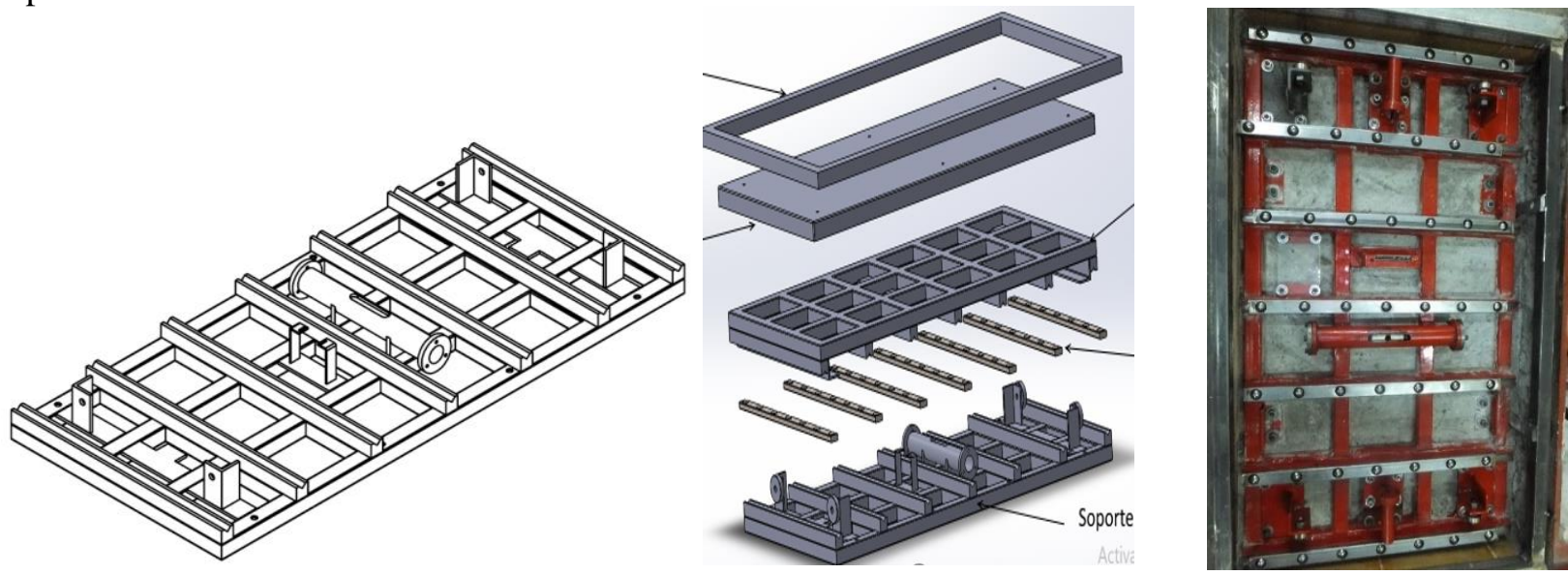

Fig: 7. Schemes of the structure of the convergence alienation device

Between the support elements of antifriction surface composed of rails antifriction observed using spheres bearing, at the top the mechanical section 1.3, movable with respect to section 1.1 is observed. In mechanical sections: bottom 1.1, 1.3 and higher, are installed the respective components associated mechanical. The assembled equipment including its upper 
platform can be seen in figure 3.33. The platform can be observed as threaded screws T required to lift and place the upper platform easily.

\section{Tests of Operation of the Equipment}

Once the equipment was assembled with all its sections: mechanical, electrical, electronic, including its control box attached to the work site, the equipment was tested under no load. Figure 8a shows the connection of the card's USB cable, with an extension to the work computer. When the power cable was connected from the computer's USB 2.0 port, $15 \mathrm{~m}$ long to the Arduino card port, Keypad shield as can be seen in figure $8 \mathrm{~b}$, then the card was activated.
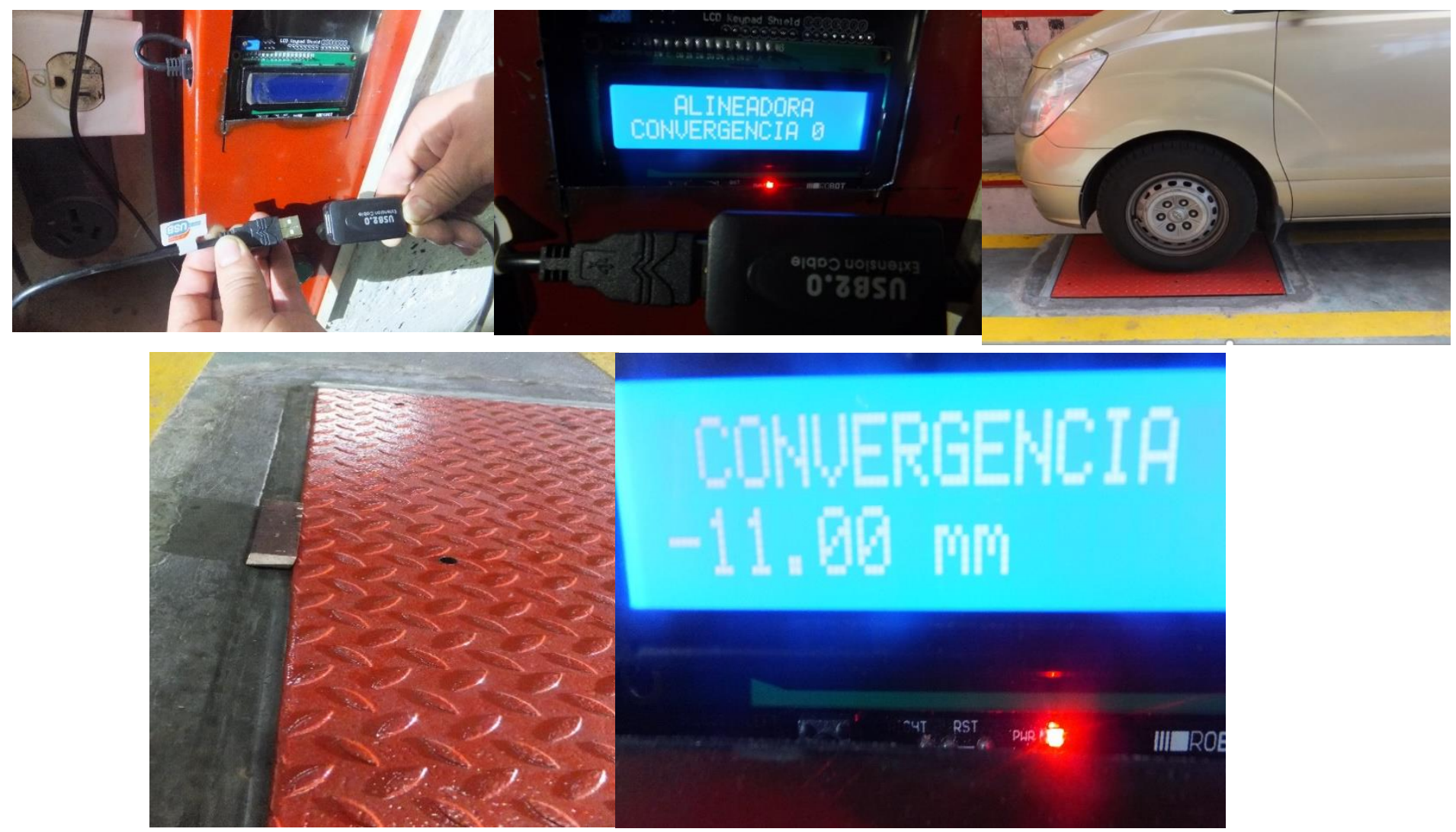

Fig. 5: Mechanical and electronic diagram

Performance tests are conducted with loaded and unloaded, then to verify proper operation of your computer compared with a comparison team teams Center Technical Review vehicle is performed. LRTV gave a reading of $2 \mathrm{~m} / \mathrm{km}$. It should be noted that the maximum permissible values for this defect are $+6.99 \mathrm{~m} / \mathrm{Km}$ for a positive convergence, and $-6.99 \mathrm{~m} /$ $\mathrm{Km}$ for a negative convergence, then the convergence diagnosis is typified as valid and the Vehicle Review is approved. The details of this test are in the public domain on the Internet. The tests carried out so far show that the equipment has sufficient vertical and transverse mechanical resistance, to the passage of light and medium-heavy vehicles of up to three tons of gross weight. The cost of the equipment is very important and the cost blocks must be determined: direct costs, indirect costs. The direct costs consider the prices of the materials of each set of pieces and the price of specific works that must be carried out on them, such as: raw material, cuts, bends, machining, perforations, welds. In a second block of direct costs, the acquisition of inputs such as: drills, cutting and grinding discs, antioxidant, paints, dye thinner is considered. A third block costs are 
considered indirect costs such as: water, electricity, rent, payment operator. Table 3.1, shown in summary the total cost per team which amounts to USD 2480, value obtained to the sum of direct costs and indirect production equipment.

\section{Conclusion}

The alignment condition of zero convergence is ideal for the smooth running of a vehicle when it is in full load operation. If the vehicle is off in terms of convergence with positive values or greater than or equal negative to 7 , it is considered a serious malfunction of the same, a situation that requires prompt restraint, which is to leave the tires at the minimum degree of convergence, or a value very close to this, in any case with convergence less than 7 in absolute value.

The deviation of the centerline of the tire from the vehicle's line of advance, which is outside the design limit of the vehicle, causes failure in the direction in which the vehicle is moving and is considered a defect of the vehicle. Since the centerline of the rim from the front line of the advance can be offset to the right or the left, it results in positive convergence and negative convergence.

Contribute to the change in the country's productive matrix, generating technology to manufacture this type of equipment locally, and with it, improve the technological level in automotive workshops nationwide, making them more competitive. It is the way the National Polytechnic School, offering technological solutions to social problems cited as concrete. It is recommended that the construction of precision elements be supervised by the author, since the lack of elements of judgment of the technical staff, undermines construction details and on several occasions the costs are implicit and affect the quality of the product.

Measure the degree of misalignment is equivalent to saying, a diagnosis of convergence in the automotive. With the help of the same equipment, it is possible to correct the convergence defect until reaching the permissible values, typified by the Vehicle Technical Review

We can calculate and conclude that the minimum safety margin in terms of the maximum load of our equipment, about the weight of the heaviest known vehicles is $40 \%$ and concerning the lightest, it is $90 \%$ and for the load that The equipment must support when working with vehicles of average weight $1000 \mathrm{Kg}$, it is $33 \%$ of the total load. These results give us assurance as to the mechanical stability of the team, even in extreme operating conditions.

Through the simulation process, it is required to verify that the stresses generated in the structure of the alignment equipment, which were previously calculated analytically, do not exceed the yield strength of the material. SAP 2000 software was used for this analysis. Simulating that the loads are applied.

\section{References}

[1] D. Hermógenes L. Martínez. Manual del Automóvil. Edit Cultural s.a. España, 2000.

[2] M. Arias. G. Paz. Manual de automóviles. 56 th Edición. Editorial Dossat. España.2006

[3] J. Rueda, Mecánica y Electrónica Automotriz. Editorial Diseli. Colombia. 2003.

[4] William F. Milliken and Douglas L. Milliken. Race Car Vehicle Dynamics. 5ta Edición, Editorial SAE International. USA. 1995, pp 245- 298.

[5] J. M. Alonso. Mecánica del Automóvil. 10ma Edición. Editorial Thomsom Paraninfo. España, 2003.

[6] W. Bolton. Mecatrónica .3ra. Edición. Editorial Alfaomega. Mexico. 2007, pp 187-256.

[7] John R. Barnes. Robust Electronic Design Reference. Book V1. Kluwer Academic Publishers, 2004.

[8] Riba, C. Selección de Materiales en el Diseño de Máquinas. Ediciones UPC. España, 2008.

[9] Livesley, R.K. Elementos finitos. 1ra edición. Editorial Limusa. México. 2008.

[11] Alfredo Roca C. Control Automático de Procesos Industriales. Editorial Díaz de Santos. España, 2014.

[12] Saturnino Soria Tello. Sistemas Automáticos Industriales. Alfaomega Editores. México, 2013.

[13] Grantham y Vincent. Sistemas de Control Moderno. Editorial Limusa. México, 1998.

[15] I. Scott Macknzie y Otros. Micro controlador 8051. 4ta. Edición. Editorial Pearson Prentice Hall. Mexico, 2007.

[16]. Budynas, Richard G. Design in Mechanical Engineering. 9na edición. Editorial Mc. Graw Hill. México, 1997 